\title{
A year in the life of biomedical research
}

As Nature Medicine celebrates one year in print, we look back over how the field of biomedical research has grown during 1995, and forward to how it might develop in this new year. Although the field of molecular medicine, and indeed the scope of articles published in Nature Medicine, is too broad to be adequately summarized here - or even easily defined - a number of areas of recent progress and future promise stand out.

Two exciting doors to future discoveries and treatment of various cancers have been opened, the one making better use of the immune system and the other starving tumors of the blood they need to grow.

\section{It was a very good year.} Although immune reactions can wreak havoc with experimental transplantation and gene therapy, it has been something of a mystery why the same immune system fails to detect and destroy cancer cells that so often express tumor-specific antigens. Recent developments have shown promise in nudging the immune system into action. Tumor-specific cytotoxic $\mathrm{T}$ lymphocytes can be induced on exposure to formalinfixed tumor samples and then exposed to the tumor in vivo, as an adoptive immunotherapy (p. 267). Also, the tumor antigen can be more efficiently presented to the cellular arm of the immune system (pp. 471; 649). This work establishes the feasibility of developing vaccines that will not only protect against new cancer but also bring about regression of existing tumors. The second notable advance in treating tumors follows the discovery that angiogenesis is key to the growth of not only the primary tumor but also distant micrometastases ( $p$. 27). Thus angiogenesis inhibitors have become a major focus for treating cancers, promising, as they do, to keep micrometastases in check by maintaining a rate of apoptosis or tumor cell death that can match that of cell proliferation (pp. 117; 149).

Gene therapy, an experimental idea ripe with overpromise, is now ready to move on to a time of more realistic aims and sober reflection (pp. 39; 1017). We know that the immune system will treat newly adopted gene products (and perhaps the viral vectors involved in delivering them) as foreign bodies and dutifully raise an aggressive immune reaction against them (p. 353). One way around this immune surveillance is to treat the patient in utero, such that the fledgling immune system will accommodate the new gene product (p. 864). Alternatively, cord blood stem cells removed immediately after birth also represent an attractive target from this point of view. In one such trial, patients receiving ex vivo transduced autologous cord blood stem cells have shown continued (adenosine deaminase) transgene expression 18 months after gene therapy (p. 1017).

Early work on transplanting fetal cells into the brains of patients suffering from neurodegenerative disorders has been thwarted by the dismal survival rate of the transplanted cells. The field is, however, enjoying a resurgence following the discovery that a variety of growth factors can significantly increase the survival and function of the transplanted cells (p. 53). Graft survival is further enhanced by limiting the oxidative stress to the graft by overexpressing free radical scavengers (pp. 201; 226). Further progress in this field will undoubtedly benefit from continued development of these possibilities, although an investigation of the root cause for the degeneration arguably deserves more attention.

The prospect, and now the practice (p. 17, this issue) of xenotransplantation has generated excitement matched by an equal measure of healthy criticism (p. 18, this issue). Recent advances have helped define the nature of the most significant barriers to the successful grafting of organs from other species into humans (pp. 403; 869), whereas others have taken important steps toward overcoming the most formidable of these barriers, the hyperacute rejection, by either limiting the runaway effects of the complement cascade (pp. 423 ; 967) or avoiding the antibody and complementdependent rejection altogether (pp. 964; 1261). This work brings closer the technical prospect of the clinically useful xenotransplant. It does not of course tackle the difficult issues surrounding the safety and ethics of using bits of other animals to treat human disease.

Finally, a series of articles have examined the challenges presented by increasingly powerful but expensive health care opportunities (pp. 719; 1104), the squeeze on research funding (pp. 14; 196) and the often difficult marriage between science and medicine (p. 281). It is clear that almost universally across biomedical research, treatment and teaching, finances dictate the rate at which the community can proceed. Although this may force an increase in efficiency, it will undoubtedly deny good researchers and doctors the opportunity to offer better treatment to the patients of today and tomorrow.

At Nature Medicine we see our role as occasionally provoking but always stimulating and fostering new thinking in all areas of the science, medicine and policy that will lead to tomorrow's medicine. In this spirit we look forward to looking back on 1996 with as much a sense of excitement as we have now.

- Barbara J. Culliton \& Adrian J. Ivinson 